\title{
Lower Extremity Arterial Calcification as a Predictor of Coronary Atherosclerosis in Patients with Peripheral Arterial Disease
}

\author{
Hwa Seon Shin, ${ }^{1}$ Mi Jung Park, ${ }^{1,}$ Kyung Nyeo Jeon, ${ }^{1}$ Jae Min Cho, ${ }^{1}$ Kyung Soo Bae, ${ }^{1}$ Dae Seob Choi, ${ }^{1}$ Jae \\ Boem Na, ${ }^{1}$ Ho Cheol Choi, ${ }^{1}$ Hye Young Choi, ${ }^{1}$ Ji Eun Kim, ${ }^{1}$ Soo Bueum Cho, ${ }^{1}$ and Sung Eun Park ${ }^{1}$ \\ ${ }^{1}$ Department of Radiology, Gyeongsang National University Hospital, Gyeongsang National University School of Medicine, Jinju, Republic of Korea \\ "Corresponding author: Mi Jung Park, Department of Radiology, Gyeongsang National University Hospital, Gyeongsang National University School of Medicine, Jinju, Republic \\ of Korea. Tel: +82-557508814, Fax: +82-557581568, E-mail: pichola@naver.com
}

Received 2015 September 16; Revised 2015 October 29; Accepted 2015 November 07.

\begin{abstract}
Background: Until now, there has been no study on the relationship between the calcification of the lower extremity arteries and significant coronary arterial disease (CAD).

Objectives: To evaluate whether lower extremity calcium scores (LECS) are associated with CAD and whether this can predict multivessel-CAD in patients with peripheral arterial disease (PAD).

Patients and Methods: We retrospectively enrolled 103 PAD patients without cardiac symptoms or known CAD. All patients underwent cardiac computed tomography (CT) and lower extremity CT within 1 month and were categorized as nonsignificant CAD, single-CAD, or multivessel-CAD. The coronary calcium scores (CCS) were quantitatively measured according to the Agatston method and LECS were semi-quantitatively measured according to the presence of lower extremity calcification in the segment. The extent of CAD was evaluated according to the presence of $\geq 50 \%$ luminal diameter stenosis in the segment of CAD.

Results: LECS in multivessel-CAD were significantly higher than those in nonsignificant CAD $(10.0 \pm 5.8$ versus $4.0 \pm 3.1, \mathrm{P}<0.001)$. LECS significantly correlated with CCS $(r=0.831, \mathrm{P}<0.001)$ and the extent of $\mathrm{CAD}(\mathrm{r}=0.631, \mathrm{P}<0.001)$. Multivariate regression analysis demonstrated LECS and log-transformed CCS were independent predictors for multivessel-CAD. In receiver operating characteristic curve analysis, the diagnostic performance of LECS was 0.807 (95\% confidence interval $=0.724-0.891, \mathrm{P}<0.001)$ for predicting multivessel-CAD.

Conclusion: Peripheral arterial calcification is significantly correlated with CAD extent in patients with PAD. Peripheral arterial calcification can be a useful marker for predicting multivessel-CAD.
\end{abstract}

Keywords: Atherosclerosis, Coronary Artery Disease, Multidetector Computed Tomography, Peripheral Arterial Disease, Vascular Calcification

\section{Background}

Peripheral arterial disease (PAD) and coronary arterial disease (CAD) both fall within the disease spectrum of atherosclerosis and PAD shares cardiovascular risk factors with CAD (1-3). Many studies have demonstrated a high prevalence of CAD in patients with PAD (4-8). Additionally, stable atherosclerotic patients without previous ischemic events have been reported to experience more cardiovascular events in cases of multiarterial disease (9). Despite these results, coronary CT angiography (CCTA) screening remains controversial for patients with PAD without known CAD or angina symptoms (10). Detection of multivessel-CAD in these patients may be important, however, as it can lead to different management strategies, specifically medical therapy or coronary intervention.

Arterial calcification represents the atherosclerotic burden of the arterial bed. Arterial calcification is an in- dependent predictor of vascular morbidity and mortality in the general population (11) and subclinical atherosclerotic disease (12). CT has been used as an effective tool to measure the calcification. In particular, coronary arterial calcification and aortic calcification measured by CT have been reported to be useful markers for predicting significant CAD and cardiovascular outcomes (13-20). Until now, there has been no study on the relationship between the calcification of the lower extremity arteries and significant CAD.

\section{Objectives}

Our study evaluated whether the extent of peripheral arterial calcification is associated with coronary calcium score (CCS) in patients with symptomatic PAD. We also evaluated whether the extent of peripheral arterial calcifica- 
tion could be useful in predicting multivessel-CAD that requires coronary intervention.

\section{Patients and Methods}

\subsection{Study Population}

From January 2007 to July 2013, 372 patients with symptomatic PAD were referred at our cardiovascular surgery department. Among them, 147 patients underwent CCTA for preoperative risk assessment for vascular surgery. We excluded the patients with chest pain $(n=3)$, known CAD $(\mathrm{n}=13)$, and suspected acute coronary syndrome $(\mathrm{n}=$ $8)$. The 16 patients were excluded because the time interval between CCTA and lower extremity CT angiography (LECTA) was more than 14 days. As it was necessary to evaluate the whole extremity artery in our study, patients with amputated lower extremities $(n=4)$ were also excluded. Hemodialysis was performed immediately after intravenous contrast injection in 7 patients with chronic renal disease to reduce contrast-induced nephrotoxicity. In the end, 103 PAD patients were included in our study. According to the Fontaine classification (21), the number of PAD patients categorized as class II (claudication), III (ischemic rest pain), and IV (ulceration, tissue loss, gangrene) were 46, 28 and 29, respectively. The hospital institutional review board approved our retrospective study and informed consent was waived. We collected the patients' data from their medical records; the baseline data included the traditional cardiovascular risk factors and laboratory findings.

\subsection{Lower Extremity CT Angiography}

LECTA was performed using a 64-detector CT (Brilliance-64, Philips Medical Systems, Best, The Netherlands). The scans covered the region from the distal abdominal aorta through the ankles. All patients received $150 \mathrm{~mL}$ of iopromide (370 mL I/mL, Ultravist 370, Bayer Shering, Berlin, Germany), administered with an injection rate of $3.5 \mathrm{~mL} / \mathrm{sec}$, which was followed by a $50 \mathrm{~mL}$ saline flush at the same rate within the region of the infrarenal abdominal aorta. LECTA parameters were: detector collimation of $64 \times 0.625 \mathrm{~mm}$, tube voltage of $120 \mathrm{kVp}$, tube current of $250 \mathrm{mAs}$, field of view (FOV) of $350 \mathrm{~mm}$, and pitch of 0.89 .

Images were transferred to an image analysis workstation (Aquarius, TeraRecon, San Mateo, California) for postprocessing. Two experienced radiologists independently evaluated all images for arterial stenosis and other abnormalities. For the initial image interpretation, the radiologist primarily used the rotating-volume maximal intensity projection. If any stenosis or calcification was found in the artery, the radiologist used the curved multiplanar reformation and the cross-sectional images for further stenosis evaluation. For analytic purposes, the peripheral arterial vascular system was divided into 3 regions and 31 segments per person (22). We divided the vascular region into three groups: aortoiliac, femoropopliteal, and below knee. The aortoiliac region included the distal aorta, common iliac arteries, and external iliac arteries. The femoropoliteal region included the deep femoral arteries, superficial femoral arteries (proximal and distal segments), and popliteal arteries (proximal and distal segments). The below-knee region included the anterior tibial arteries (proximal and distal segments), tibiofibular trunks, posterior tibial arteries (proximal and distal segments), and peroneal arteries (proximal and distal segments).

Each LECTA segment was evaluated for the presence and degree of arterial stenosis. The degree of arterial stenosis in each segment was defined as insignificant $(<50 \%$ luminal diameter narrowing) or significant stenosis ( $\geq$ $50 \%$ luminal diameter narrowing). If coexisting arterial stenoses were present in a single segment, the most severe stenotic lesion was evaluated. Lower extremity arterial index (LEAI) was defined as the sum of the segments with significant stenosis in the lower extremity arteries. The radiologist also recorded the presence of vessel wall calcifications. It is difficult to differentiate between tiny calcified plaques and the contrast-enhanced lumen in LECTA, so the presence of calcification in a segment was scored as 0 (absence of calcification or calcified plaque $<3 \mathrm{~mm}$ in length) or 1 (calcified plaque $\geq 3 \mathrm{~mm}$ in diameter) (22). The lower extremity calcium score was measured by the sum of the individual scores from segments with calcified plaque and reported as LECS. Finally, we measured the LEAI and LECS in all segments and vascular regions. Figure $1 \mathrm{~A}$ shows a representative case that has a significant stenotic lesion in LECTA.

\subsection{Coronary CT Angiography}

CCTA was performed within 14 days after LECTA using the same 64-MDCT scanner. Patients with an heart rate $>$ 70 bpm received a long-acting beta-blocker (40 mg propranolol hydrochloride, Pranol @: Daewoong, Seoul, Korea) orally 1 hour before CCTA. Patients had prospectively undergone ECG-gated unenhanced CT for coronary calcium scoring by the method of Agatston et al. (23). Calcium scoring scans were performed at a tube voltage of $120 \mathrm{kVp}$, effective tube current of $55 \mathrm{mAs}$, slice collimation of $40 \times 0.625$ $\mathrm{mm}$, gantry rotation time of 0.4 second, and FOV of 220 $\mathrm{mm}$. The images were reconstructed with a section thickness of $3 \mathrm{~mm}$, increments of $3 \mathrm{~mm}$, and a medium smooth 
reconstruction filter (Philips "B" filter). The Agatston coronary calcium score (CCS) was measured using semiautomatic software (HeartBeat CS, Philips Medical Systems).

Just before the injection of contrast material, $0.6 \mathrm{mg}$ of nitroglycerin was administered sublingually for vessel dilatation. All patients received $100 \mathrm{~mL}$ of iomeprol (400 $\mathrm{mL} \mathrm{I/mL,} \mathrm{Iomeron} \mathrm{400;} \mathrm{Bracco,} \mathrm{Milan,} \mathrm{Italy),} \mathrm{administered}$ at an injection rate of $5 \mathrm{~mL} / \mathrm{sec}$, which as followed by a 30 $\mathrm{mL}$ saline flush at the same rate. Acquisition was started immediately at a threshold of $150 \mathrm{HU}$ within the region of interest in the descending aorta.

Coronary CT angiography was performed at a tube voltage of $120 \mathrm{kVp}$, with an effective tube current of 800 to $1,000 \mathrm{mAs}$ (depending on the BMI), slice collimation of 64 $\times 0.625 \mathrm{~mm}$ acquisition, gantry rotation time of $0.4 \mathrm{sec}-$ ond, FOV of $220 \mathrm{~mm}$ and pitch of 0.2. The mean effective radiation dose was calculated to be $11.2 \pm 2.4$. The images were reconstructed with a reconstructed section thickness of $0.8 \mathrm{~mm}$, reconstruction increment of $0.4 \mathrm{~mm}$, and a cardiac standard reconstruction filter (Philips “CB" filter).

The multiphasic reconstructions of CCTA were performed at $35 \%, 40 \%, 45 \%, 55 \%, 65 \%, 75 \%, 85 \%$, and $95 \%$ of the $\mathrm{R}-\mathrm{R}$ interval. The reconstruction intervals with the fewest motion artifacts were selected for further diagnosis. The CCTA image was interpreted by one experienced reader, who was blinded to all patient characteristics and LECTA results. The CCTA images were evaluated on an image analysis workstation (Aquarius, TeraRecon, San Mateo, California) for postprocessing. For image interpretation, the radiologist primarily used the curved multiplanar reformation and maximum intensity projection. If any lesions with calcified plaque were found in the coronary artery, the radiologist further analyzed the cross-sectional images of the lesion. The data were evaluated for the presence of significant coronary artery stenosis within all 17 coronary segments (24). Significant CAD was determined by $\geq 50 \%$ luminal diameter stenosis. Multivessel-CAD included twovessel CAD, three-vessel CAD, and CAD involving the left main coronary artery. The coronary artery index (CAI) was defined as the sum of all segments with significant stenosis in the coronary artery (Figure 1B).

If any lesion with $\geq 50 \%$ stenosis was observed in the coronary artery or it was impossible to measure the degree of coronary artery stenosis because of blooming and motion artifacts, invasive coronary angiography (ALLURA Xper FD 20, Philips, Netherland) was performed for further diagnosis and management. One experienced cardiologist measured the severity of coronary stenoses with visual estimation in two orthogonal views and matched the lesion with MDCT results (Figures $1 C$ and 1D). If there was any disagreement between invasive coronary angiography and MDCT regarding the degree of stenosis, it was primar- ily determined by invasive coronary angiography. As the CCTA has high negative predictive value for the diagnosis of significant CAD, no additional study was performed in CAD with $<50 \%$ stenosis.

\subsection{Statistical Analysis}

The Kolmogorov-Smirnov test was used to analyze data normality. Normally distributed continuous variables were expressed as the mean $\pm \mathrm{SD}$, and non-normally distributed continuous variables were expressed as the median value (interquartile range). Differences between the two groups of continuous variables were analyzed using unpaired t-tests. Differences between the two groups of discontinuous variables were analyzed using the $\chi^{2}$ test. As CCS was not normally distributed and showed highly positive skewness, the log transformation value was performed for further analyses. The Pearson correlation coefficient was employed to determine the correlation between the $\log$ transformation of CCS (log CCS), LECS, and CAI. Univariate binary logistic regression analysis was performed and the variables with $\mathrm{P}<0.20$ were then entered into a forward stepwise multivariate logistic regression model to assess the independent predictors for multivessel-CAD. Multivariate binary logistic regression analysis was performed to assess the independent predictors of multivessel-CAD. Receiver operating characteristic (ROC) analysis was employed to determine sensitivity, specificity, and positive and negative predictive values of LECS in detecting multivessel CAD. Optimal cutoffs were determined by the maximum Youden index. From the ROC analysis, the sensitivity and specificity of both log CCS and LECS were extracted for the prediction of multivessel-CAD. Statistical significance was considered as $\mathrm{P}<0.05$ (two-tailed). All statistical analyses were performed using SPSS 21.0 (IBM-SPSS, Chicago, IL, USA).

\section{Results}

\subsection{Patient Characteristics}

Of the 103 symptomatic patients with PAD, 93 (90.3\%) were male and the mean age was $67.5 \pm 8.5$ years (range, 45 - 88 years). Patients were classified as nonsignificant CAD $(\mathrm{n}=45)$, single-CAD $(\mathrm{n}=26)$, and multivessel-CAD $(\mathrm{n}=32)$. There was no significant difference in the cardiovascular risk factors among the three groups (Table 1).

4.2. Association of Lower Extremity Artery Disease, Lower Extremity Artery Calcification, and Coronary Artery Calcification with Coronary Artery Disease

We evaluated 3,193 arterial segments in the lower extremity arteries using LECTA. Lower extremity arterial le- 

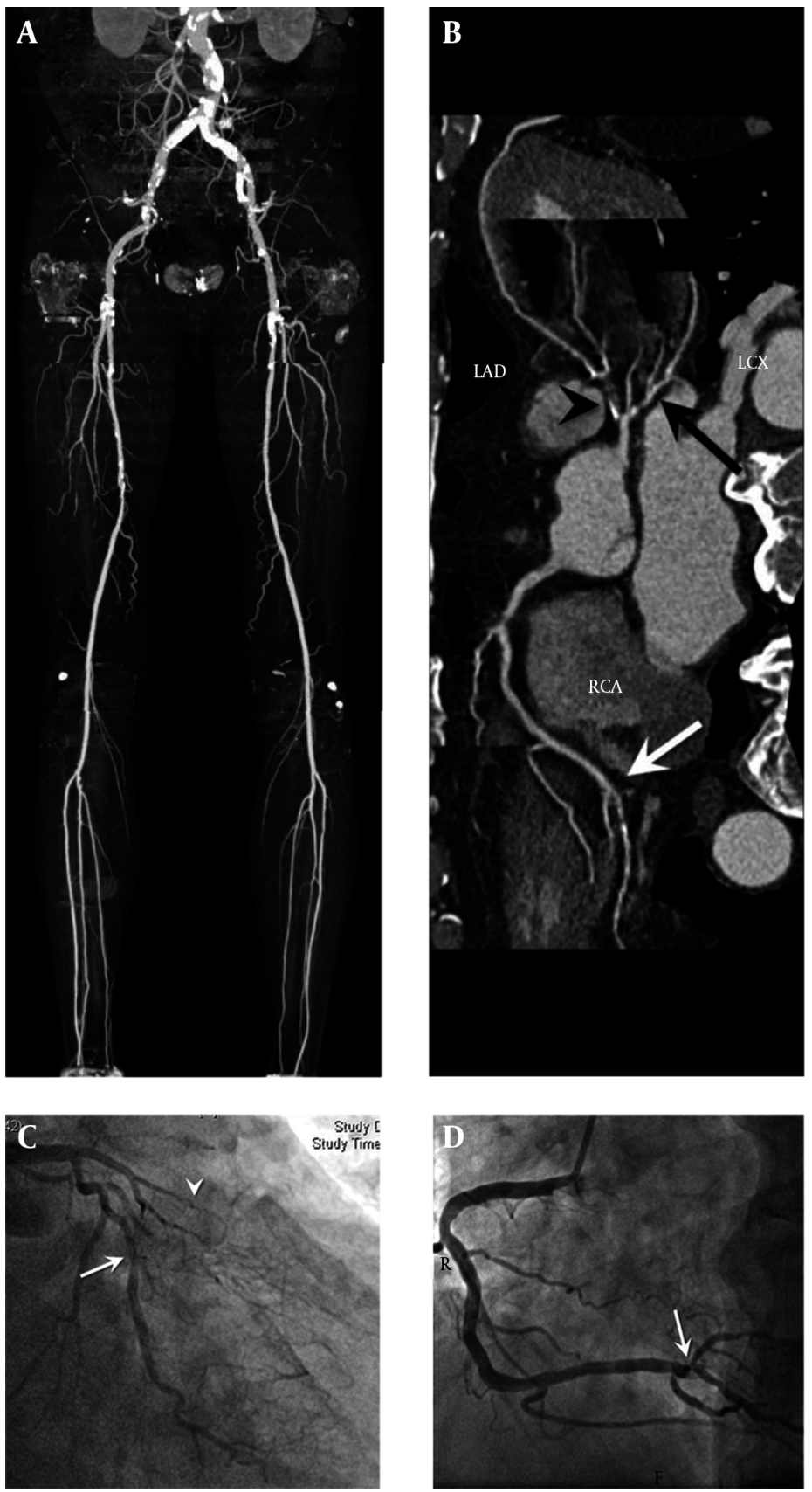

Figure 1. A 74 year-old male patient with claudication underwent lower extremity CT angiography. Lower extremity calcium was detected in the distal aorta, both common iliac arteries, both external iliac arteries, both common femoral arteries, both proximal superficial femoral arteries, right distal superficial femoral arteries, and right popliteal artery. A, The lower extremity calcium score was 11. B, Coronary CT angiography CCTA was performed and the coronary calcium score was 315.52. C, Invasive coronary angiography showed chronic total occlusion of the left anterior descending artery (arrowhead) and severe stenosis of the proximal left circumflex artery (arrow). D, Severe stenosis in trifurcation site of distal RCA (arrow). The stenotic lesions observed by invasive angiography were matched with CCTA results.

sions with significant stenosis were detected in 667 segments (20.9\%). We evaluated 1,751 arterial segments in coronary arteries using CCTA. Coronary arterial lesions with significant stenosis were detected in 149 (8.5\%) segments. LEAI was significantly higher in multivessel-CAD than in nonsignificant CAD (Table 2). LEAI in all segments was weakly correlated with the extent of $\operatorname{CAD}(\mathrm{r}=0.432, \mathrm{P}<0.001)$.

The median CCS and LECS in patients with PAD were 
Table 1. Demographic and Clinical Characteristics Among Nonsignificant CAD (Coronary Artery Disease), Single-CAD, and Multivessel-CAD Patients With Peripheral Artery Disease $^{\mathrm{a}}$

\begin{tabular}{|c|c|c|c|c|}
\hline Variable & Nonsignificant CAD $(n=45)$ & Single-CAD $(n=26)$ & Multivessel-CAD ( $n=32)$ & P Value \\
\hline Age, $y$ & $66.4 \pm 8.9$ & $68.8 \pm 7.7$ & $68.1 \pm 7.3$ & 0.437 \\
\hline Gender, Male & $42(93.3)$ & $22(84.6)$ & $29(90.6)$ & 0.488 \\
\hline Height, $\mathrm{cm}$ & $163.4 \pm 6.2$ & $165.5 \pm 8.3$ & $164.9 \pm 7.7$ & 0.472 \\
\hline Weight, kg & $56.9 \pm 8.1$ & $57.5 \pm 10.3$ & $56.9 \pm 9.9$ & 0.959 \\
\hline Body mass index, $\mathrm{kg} / \mathrm{m}^{2}$ & $21.2 \pm 2.4$ & $21.0 \pm 3.2$ & $20.8 \pm 3.0$ & 0.820 \\
\hline Smoking, pack-years & $20.0 \pm 17.3$ & $26.3 \pm 23.0$ & $34.4 \pm 24.3$ & 0.033 \\
\hline Diabetes Mellitus & $13(28.9)$ & $14(53.8)$ & $14(43.8)$ & 0.101 \\
\hline Hypertension & $20(44.4)$ & $15(57.7)$ & $20(62.5)$ & 0.063 \\
\hline Cerebral disease & $7(15.6)$ & $10(14.1)$ & $5(15.6)$ & 0.980 \\
\hline Chronic renal disease & $2(4.4)$ & $1(3.8)$ & $4(12.5)$ & 0.129 \\
\hline Total cholesterol, mmol/L & $145.6 \pm 45.2$ & $156.6 \pm 45.6$ & $164.1 \pm 44.5$ & 0.223 \\
\hline HDL-cholesterol, mmol/L & $46.1 \pm 14.8$ & $41.3 \pm 10.5$ & $40.3 \pm 16.4$ & 0.611 \\
\hline LDL-cholesterol, mmol/L & $84.1 \pm 28.8$ & $87.7 \pm 21.2$ & $95.5 \pm 39.8$ & 0.476 \\
\hline Triglyceride, mmol/L & $122.8 \pm 108.0$ & $205.7 \pm 135.5$ & $211.1 \pm 120.4$ & 0.424 \\
\hline Glucose, mmol/L & $134.8 \pm 50.1$ & $138.7 \pm 59.7$ & $139.7 \pm 60.8$ & 0.648 \\
\hline Creatinine, $\mu \mathrm{mol} / \mathrm{L}$ & $0.95 \pm 0.57$ & $1.38 \pm 1.26$ & $1.91 \pm 1.72$ & 0.191 \\
\hline C-reactive protein & $43.6 \pm 45.1$ & $33.0 \pm 48.0$ & $47.8 \pm 55.1$ & 0.514 \\
\hline Fontain II/III/IV, n & $24 / 14 / 7$ & $10 / 6 / 10$ & $10 / 12 / 10$ & 0.136 \\
\hline
\end{tabular}

${ }^{\mathrm{a}}$ values are expressed as No. (\%) or mean $\pm \mathrm{SD}$.

Table 2. Comparison of CT Characteristics Among Nonsignificant CAD, Single-CAD, and Multivessel-CAD ${ }^{\mathrm{a}, \mathrm{b}, \mathrm{c}}$

\begin{tabular}{|c|c|c|c|c|}
\hline Variable & Nonsignificant $\operatorname{CAD}(n=45)$ & Single-CAD $(n=26)$ & Multivessel-CAD $(\mathbf{n}=32)$ & P Value \\
\hline Lower extremity arterial index (LEAI) & $5.2 \pm 3.5$ & $8.0 \pm 4.2$ & $9.0 \pm 5.0^{b}$ & $<0.001$ \\
\hline Aortoiliac & $1.2 \pm 1.5$ & $1.2 \pm 1.5$ & $1.7 \pm 1.8$ & 0.370 \\
\hline Femoropopliteal & $2.03 \pm 2.1$ & $2.3 \pm 2.9$ & $3.2 \pm 2.3$ & 0.127 \\
\hline Below knee & $2.0 \pm 2.5$ & $4.7 \pm 3.3^{\mathrm{b}}$ & $4.5 \pm 3.5^{\mathrm{b}}$ & $<0.001$ \\
\hline Lower extremity calcium score (LECS) & $3.9 \pm 2.3$ & $5.7 \pm 2.9$ & $9.3 \pm 4.2^{\mathrm{b}, \mathrm{c}}$ & $<0.001$ \\
\hline Aortoiliac & $1.4 \pm 1.5$ & $1.7 \pm 1.7$ & $1.6 \pm 1.8$ & 0.789 \\
\hline Femoropopliteal & $1.2 \pm 1.5$ & $1.6 \pm 2.4$ & $2.5 \pm 2.1^{\mathrm{b}}$ & 0.028 \\
\hline Below knee & $1.2 \pm 1.7$ & $2.4 \pm 2.3$ & $5.2 \pm 4.1^{\mathrm{b}, \mathrm{c}}$ & $<0.001$ \\
\hline
\end{tabular}

${ }^{\mathrm{a}}$ Values are expressed as mean $\pm \mathrm{SD}$.

${ }^{\mathrm{b}} \mathrm{P}<0.05$ compared to nonsignificant CAD.

${ }^{\mathrm{C}} \mathrm{P}<0.05$ compared to single-CAD.

$318.0 \pm 954.3$ (from 0 to 3750.1 ) and $5.0 \pm 3.9$ (from 0 to 18 ), respectively. Calcification of the coronary artery and the lower extremity arteries was detected in $87.4 \%(n=90)$ and 95.1\% ( $n=98)$ of those patients, respectively. CCS was significantly different in the three groups $(234.3 \pm 391.3$ in nonsignificant CAD, $588.8 \pm 811.0$ in single-CAD, and 1,741.1 \pm
1,475.5 in multivessel-CAD, $\mathrm{P}<0.001)$. In per segment analysis, lower extremity arterial lesions with calcification were detected in 681 segments (21.3\%). Some 5 patients without lower extremity arterial calcification notably had evidence of neither calcification of the coronary artery nor significant CAD. LECS in all segments was significantly higher in 
multivessel-CAD than in nonsignificant CAD (Table 2). LECS in all segments was significantly correlated with CCS $(\mathrm{r}=$ $0.831, \mathrm{P}<0.001)$ and $\mathrm{CAI}(\mathrm{r}=0.631, \mathrm{P}<0.001)$.

Furthermore, we classified LEAI and LECS according to vascular region of lower extremity arteries and compared those variables among three CAD groups (Table 2). LECS and LEAI in the below-knee region were significantly higher in multivessel-CAD than in nonsignificant CAD. On the other hand, LECS and LEAI in the aortoiliac region showed no significant difference among the three groups.

4.3. Ability of Lower Extremity Artery Calcification, Extent of Lower Extremity Arterial Disease, And Coronary Artery Calcifcation to Predict Multivessel Coronary Artery Disease

In patients with $\mathrm{PAD}$, simple binary logistic regression analysis revealed that age (1.009, 95\% CI; $1.003-1.015)$, BMI (0.968, 95\% CI; 0.949 - 0.987), HDL-cholesterol (0.953, 95\% CI; 0.911 - 0.993), LDL-cholesterol (1.021, 95\% CI; 0.998 - 1.042), Fontain IV (1.761; 95\% CI; 0.726 - 4.979), LEAI in all segments (1.086, 95\% CI; 0.971 - 1.175), LECS in all segments (1.633, 95\% CI; 1.349 - 1.977), and log CCS (14.316, 95\% CI; 4.657 - 44.009) showed a trend $(\mathrm{P}<0.20)$ toward an association with multivessel-CAD. All these variables were entered into a forward stepwise multivariate binary logistic regression model. Multivariate regression analysis demonstrated that LECS in all segments (1.439, 95\% CI;1.072 -1.930) and $\log$ CCS (8.288, 95\% CI; 2.317 - 29.645) were independent predictors of multivessel-CAD (Table 3 ).

The diagnostic performance of LECS in all segments, LEAI in all segments, and log CCS for differentiating the patients with multivessel-CAD was assessed by ROC curve analysis (Figure 2). The best cutoff value for LECS in all segments was $\geq 6$ (sensitivity $=71.9 \%$, specificity $=67.6 \%)$ and the area under the curve was 0.820 (95\% CI=0.734 to 0.907; $\mathrm{P}<0.001)$. The best cutoff value of LEAI in all segments was $\geq 8$ (sensitivity $=62.5 \%$, specificity $=57.7 \%$ ) and the area under the curve was 0.661 ( $95 \% \mathrm{CI}=0.551$ to $0.771 ; \mathrm{P}=0.009$ ). The best cutoff value of $\log$ CCS was $\geq 2.66$ (sensitivity = $81.3 \%$, specificity $=78.9 \%$ ) and the area under the curve of $\log$ CCS was 0.865 (95\% CI = 0.792 to 0.938; $\mathrm{P}<0.001$ ).

We also assessed the diagnostic performance of LECS and LEAI in the below-knee region to differentiate the patients with multivessel-CAD. The best cutoff value of LECS in the below-knee region was $\geq 4$ (sensitivity $=68.8 \%$, specificity $=84.5 \%)$ and the area under the curve was $0.722(95 \%$ $\mathrm{CI}=0.597$ to $0.846 ; \mathrm{P}<0.001)$. The best cutoff value of $\log$ CCS was $\geq 2.66$ (sensitivity $=81.3 \%$, specificity $=78.9 \%$ ) and the area under the curve of log CCS was 0.865 (95\% CI = 0.792 to $0.938 ; \mathrm{P}<0.001$ ).

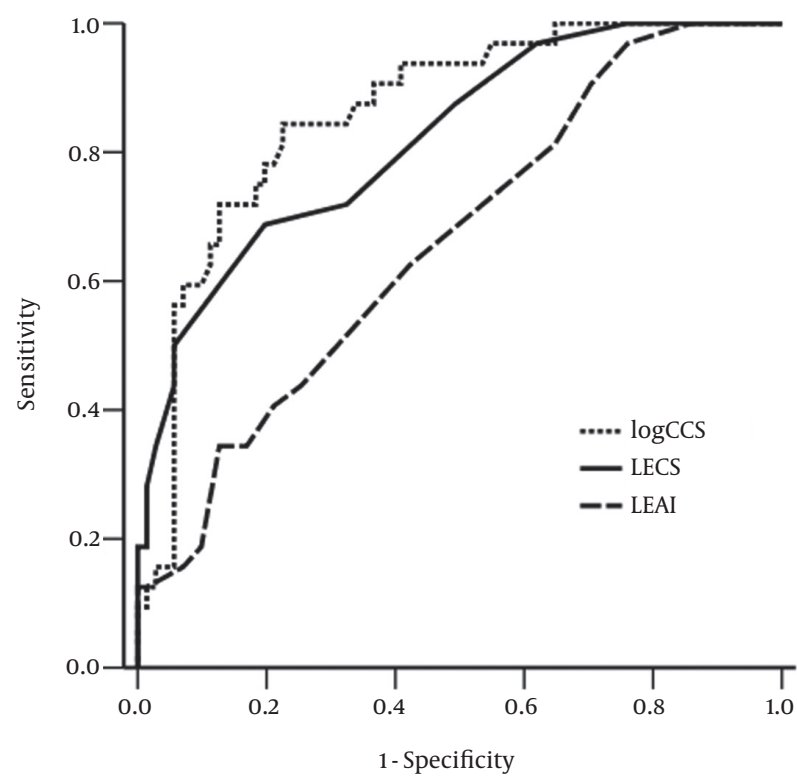

Figure 2. Receiver operator characteristic curves of log-transformed coronary calcium score (log CCS), lower extremity arterial index (LEAI), and lower extremity calcium score (LECS) in all segments, showing the diagnostic performance for the prediction of multivessel-coronary artery disease. The area under the curve of LECS in all segments, that of LEAI, and that of CCS were 0.820 (95\% confidence interval $=0.734$ to $0.907 ; \mathrm{P}<0.001), 0.661(95 \% \mathrm{CI}=0.551$ to $0.771 ; \mathrm{P}=0.009)$ and 0.865 (95\% confidence interval $=0.792$ to $0.938 ; \mathrm{P}<0.001)$, respectively.

\section{Discussion}

In the present study, we investigated the possible role and clinical implication of LECS association with CAD in patients with symptomatic PAD. We demonstrated that PAD patients with multivessel CAD had significantly higher LECS than those with nonsignificant CAD. Furthermore, LECS in LECTA was a significant predictor for the diagnosis of multivessel-CAD.

PAD is a systemic atherosclerotic disease and a high global risk factor for CAD (1-3). We therefore investigated the relationships among the extent of PAD, the extent of CAD, LECS, and CCS in patients with PAD. CAI had a strong correlation with LECS compared to LEAI in our study. LECS and CCS were predictors for multivessel-CAD in our study. There has been no report demonstrating a direct relationship between LECS and the extent of CAD in patients with PAD. Previous studies, however, did demonstrate that the presence of peripheral arterial calcification was strongly associated with cardiovascular mortality in Monckeberg's arteriosclerosis (25), diabetes (26), and end-stage renal disease (27). Calcification does not play a major role in inducing vascular stenosis in the early atherosclerotic stages, but increased arterial stiffness may contribute vas- 
Table 3. Logistic Binary Regression Analysis for Predicting Multivessel-Coronary Arterial Disease in Patients With Peripheral Arterial Disease

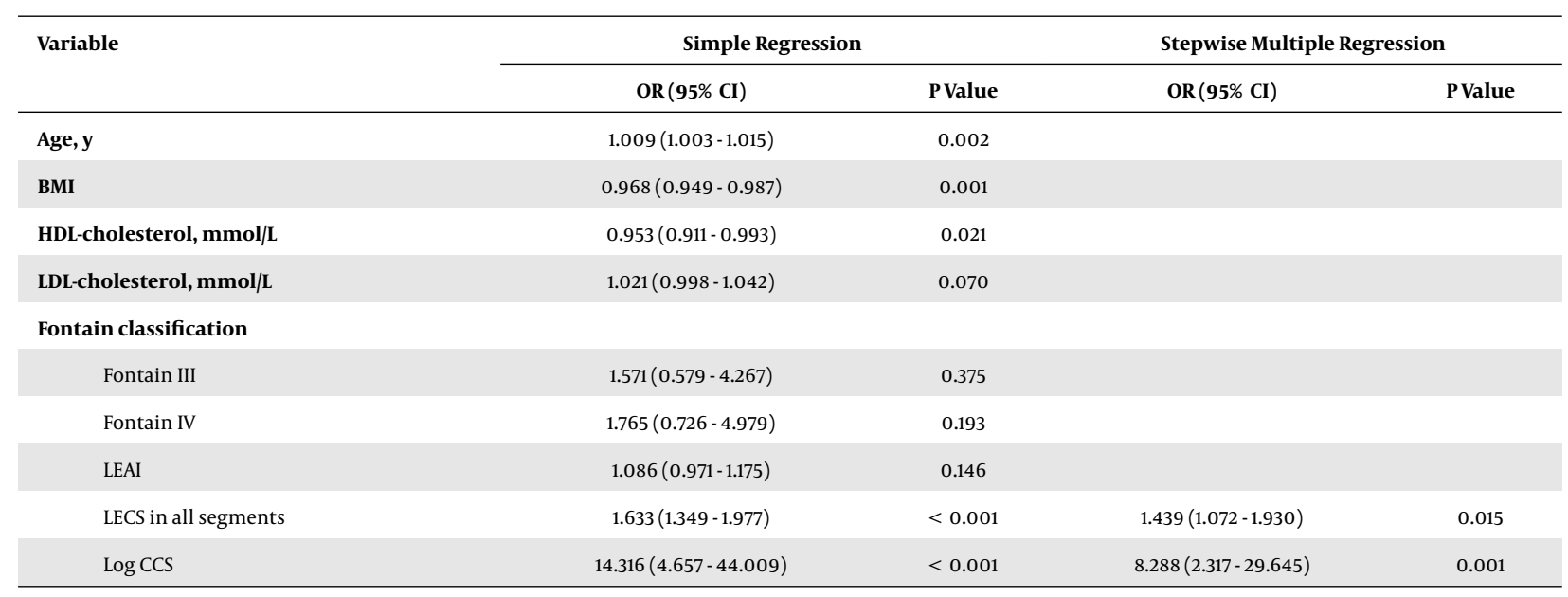

Abbreviation: CI, confidence interval; BMI, body mass index; CCS, coronary calcium score; LEAI, lower extremity arterial index; LECS, lower extremity calcium score; OR, odds ratio.

cular calcification and subsequently stenosis in the later atherosclerotic stages (28). That is why we enrolled symptomatic patients with PAD, which indicates advanced PAD. Patients with peripheral arterial calcification were therefore included in our study. Previous studies demonstrated that increased arterial stiffness causes aortic calcification and adverse cardiovascular events in patients with PAD (2931).

We further investigated the difference of LEAI, LECS, and CAD in relation to vascular regions. In our study, LEAI and LECS in the below-knee region were significantly higher in multivessel-CAD rather than in nonsignificant CAD. Our study, however, showed that the diagnostic performance of LEAI and LECS in the below-knee region were relatively lower than in all segments. In other words, LEAI and LECS in the below-knee region were weakly associated with $\mathrm{CAD}$; that is, the diagnostic impact of those variables was weak. PAD is a heterogeneous disease group and the disease location and distribution of PAD is important when predicting mortality. A previous study showed CAD was associated with LEAI in the femoropopliteal and belowknee regions, even though LEAI of the below-knee region was less associated with CAD (32). This study also showed that older age, being male, having diabetes, and having critical limb ischemia were strongly associated with LEAI in the below-knee region (32), so those variables could be confounding factors of our study.

We further investigated whether calcification and severity of the lower extremity arteries could predict multivessel-CAD. Until now, there have been no specific clinical trials to investigate the survival benefit in those patients. Coronary revascularization, however, has been known to be superior to medical therapy alone for reducing cardiovascular events in multivessel-CAD $(33,34)$. The presence of multivessel-CAD may therefore be useful when determining a further treatment plan in patients with PAD. Our study showed that if calcifications were observed in more than six segments of the lower extremity arteries, there was a high probability of multivessel-CAD. Traditionally, the coronary calcium score has been a widely accepted indicator of significant CAD and cardiovascular outcomes $(14,16-18,20)$. The previous study notably reported that increased CCS was associated with an increased frequency of multi-vessel CAD (35). In our study, the diagnostic performance of LECS in all segments for the prediction of multivessel-CAD was relatively higher than that of LEAI and slightly lower than that of CCS. These findings suggest that we could predict multivessel-CAD using LECT alone, without additional radiation exposure, but LECS could not replace CCS in the prediction of multivessel-CAD.

We acknowledge several limitations in our study. First, our study had a retrospective design and included a relatively small number of patients. Additionally, well-known cardiovascular risk factors, such as diabetes, chronic renal disease, and hypertension, were not controlled in this study, so the results may be biased. Second, calcifications in the lower extremity arteries were not measured quantitatively, as with calcium volume or Agatston scores, because there was no pre-enhanced image for LECT in our study. Furthermore, calcified plaques with lower density of calcium could be missed. Automatic quantitative analysis in a pre-enhanced CT scan is a more accurate and reproducible method for measuring the amount of calcification. Third, hemodynamically significant CAD, rather 
than multivessel-CAD, would be more important for determining a treatment plan and predicting the cardiovascular outcome in patients with CAD. The stress perfusion image might be useful to identify the predictors of cardiovascular outcomes in patients with PAD.

In conclusion, LECS is associated with CCS and CAD. LECS measured by LECTA might be a useful marker for predicting multivessel-CAD in patients with PAD.

\section{Footnote}

Authors' Contributions: Study concept and design: Mi Jung Park and Hwa Seon Shin; acquisition of data: Mi Jung Park and Kyung Nyeo Jeon; analysis and interpretation of data: Mi Jung Park and Ho Cheol Choi; drafting of the manuscript: Hwa Seon Shin, Jae Min Cho and Sung Eun Park; critical revision of the manuscript for important intellectual content: Mi Jung Park, Kyung Soo Bae and Soo Bueum Cho; statistical analysis: Hye Young Choi, Ji-Eun Kim and Ho Cheol Choi; administrative, technical, and material support: Dae Seob Choi and Jae Boem Na; study supervision: Mi Jung Park

\section{References}

1. Poredos P, Jug B. The prevalence of peripheral arterial disease in high risk subjects and coronary or cerebrovascular patients. Angiology. 2007;58(3):309-15. doi: 10.1177/0003319707302494. [PubMed: 17626985].

2. Bhatt DL, Steg PG, Ohman EM, Hirsch AT, Ikeda Y, Mas JL, et al International prevalence, recognition, and treatment of cardiovascular risk factors in outpatients with atherothrombosis. JAMA. 2006;295(2):180-9. doi: 10.1001/jama.295.2.180. [PubMed: 16403930].

3. Lange S, Trampisch HJ, Haberl R, Darius H, Pittrow D, Schuster A, et al. Excess 1-year cardiovascular risk in elderly primary care patients with a low ankle-brachial index (ABI) and high homocysteine level. Atherosclerosis. 2005;178(2):351-7. doi: 10.1016/j.atherosclerosis.2004.09.003. [PubMed: 15694945].

4. Criqui MH, Langer RD, Fronek A, Feigelson HS, Klauber MR, McCann TJ, et al. Mortality over a period of 10 years in patients with peripheral arterial disease. $N$ Engl J Med. 1992;326(6):381-6. doi: 10.1056/NEJM199202063260605. [PubMed: 1729621].

5. Hertzer NR, Beven EG, Young JR, O'Hara PJ, Ruschhaupt W3, Graor RA, et al. Coronary artery disease in peripheral vascular patients. A classification of 1000 coronary angiograms and results of surgical management. Ann Surg. 1984;199(2):223-33. [PubMed: 6696538].

6. Bridges $\mathrm{AB}$, Kennedy NSJ, Pringle $\mathrm{TH}$, et al. Prediction of late cardiac events by dipyridamole thallium scintigraphy in patients with intermittent claudication and occult coronary artery disease. Am J Cardiol. 1996;78(7):736-40. doi:10.1016/S0002-9149(96)00412-2.

7. Duran NE, Duran I, Gurel E, Gunduz S, Gol G, Biteker M, et al. Coronary artery disease in patients with peripheral artery disease. Heart Lung. 2010;39(2):116-20. doi: 10.1016/j.hrtlng.2009.07.004. [PubMed: 20207271].

8. Sukhija R, Yalamanchili K, Aronow WS. Prevalence of left main coronary artery disease, of three-or four-vessel coronary artery disease, and of obstructive coronary artery disease in patients with and without peripheral arterial disease undergoing coronary angiography for suspected coronary artery disease. Am JCardiol. 2003;92(3):304-5. doi: 10.1016/S0002-9149(03)00632-5.

9. Bhatt DL, Eagle KA, Ohman EM, Hirsch AT, Goto S, Mahoney EM, et al. Comparative determinants of 4-year cardiovascular event rates in stable outpatients at risk of or with atherothrombosis. JAMA. 2010;304(12):1350-7. doi: 10.1001/jama.2010.1322. [PubMed: 20805624].

10. European Stroke O, Tendera M, Aboyans V, Bartelink ML, Baumgartner I, Clement D, et al. ESC Guidelines on the diagnosis and treatment of peripheral artery diseases: Document covering atherosclerotic disease of extracranial carotid and vertebral, mesenteric, renal, upper and lower extremity arteries: the Task Force on the Diagnosis and Treatment of Peripheral Artery Diseases of the European Society of Cardiology (ESC). Eur Heart J. 2011;32(22):2851-906. doi: 10.1093/eurheartj/ehr211. [PubMed: 21873417].

11. Rennenberg RJ, Kessels AG, Schurgers LJ, van Engelshoven JM, de Leeuw PW, Kroon AA. Vascular calcifications as a marker of increased cardiovascular risk: a meta-analysis. Vasc Health Risk Manag 2009;5(1):185-97. [PubMed: 19436645].

12. Wong ND, Lopez VA, Allison M, Detrano RC, Blumenthal RS, Folsom AR, et al. Abdominal aortic calcium and multi-site atherosclerosis: the Multiethnic Study of Atherosclerosis. Atherosclerosis. 2011;214(2):43641. doi: 10.1016/j.atherosclerosis.2010.09.011. [PubMed: 21035803].

13. Lee HY, Song IS, Yoo SM, Rho JY, Moon JY, White CS. Can the extent of epicardial adipose tissue thickness or the presence of descending thoracic aortic calcification predict significant coronary artery stenosis in patients with a zero coronary calcium score on multi-detector CT?. Atherosclerosis. 2010;212(2):495-500. doi: 10.1016/j.atherosclerosis.2010.01.047. [PubMed: 20189570].

14. Detrano R, Guerci AD, Carr JJ, Bild DE, Burke G, Folsom AR, et al Coronary calcium as a predictor of coronary events in four racial or ethnic groups. N Engl J Med. 2008;358(13):1336-45. doi: 10.1056/NEJMoa072100. [PubMed: 18367736].

15. Budoff MJ, Nasir K, Katz R, Takasu J, Carr JJ, Wong ND, et al. Thoracic aortic calcification and coronary heart disease events: the multi-ethnic study of atherosclerosis (MESA). Atherosclerosis. 2011;215(1):196-202. doi: 10.1016/j.atherosclerosis.2010.11.017. [PubMed: 21227418].

16. Budoff MJ, Shaw LJ, Liu ST, Weinstein SR, Mosler TP, Tseng PH, et al. Long-term prognosis associated with coronary calcification observations from a registry of 25,253 patients. J Am Coll Cardiol. 2007;49(18):1860-70. doi: 10.1016/j.jacc.2006.10.079. [PubMed: 17481445].

17. Nasir K, Shaw LJ, Liu ST, Weinstein SR, Mosler TR, Flores PR, et al. Ethnic differences in the prognostic value of coronary artery calcification for all-cause mortality. J Am Coll Cardiol. 2007;50(10):953-60. doi: 10.1016/j.jacc.2007.03.066. [PubMed: 17765122].

18. Shaw LJ, Raggi P, Schisterman E, Berman DS, Callister TQ. Prognostic value of cardiac risk factors and coronary artery calcium screening for all-cause mortality. Radiology. 2003;228(3):826-33. doi: 10.1148/radiol.2283021006. [PubMed: 12869688].

19. Wilson PW, Kauppila LI, O’Donnell CJ, Kiel DP, Hannan M, Polak JM, et al. Abdominal aortic calcific deposits are an important predictor of vascular morbidity and mortality. Circulation. 2001;103(11):1529-34 [PubMed: 11257080]

20. Arad Y, Goodman KJ, Roth M, Newstein D, Guerci AD. Coronary calcification, coronary disease risk factors, C-reactive protein, and atherosclerotic cardiovascular disease events: the St. Francis Heart Study. J Am Coll Cardiol. 2005;46(1):158-65. doi: 10.1016/j.jacc.2005.02.088. [PubMed: 15992651].

21. Fontaine R, Kim M, Kieny R. [Surgical treatment of peripheral circulation disorders]. Helv Chir Acta. 1954;21(5-6):499-533. [PubMed: 14366554]. 
22. Ouwendijk R, Kock MC, van Dijk LC, van Sambeek MR, Stijnen T, Hunink MG. Vessel wall calcifications at multi-detector row CT angiography in patients with peripheral arterial disease: effect on clinical utility and clinical predictors. Radiology. 2006;241(2):603-8. doi: 10.1148/radiol.2412050781. [PubMed: 16966479].

23. Agatston AS, Janowitz WR, Hildner FJ, Zusmer NR, Viamonte M, Detrano R. Quantification of coronary artery calcium using ultrafast computed tomography. J Am Coll Cardiol. 1990;15(4):827-32. doi 10.1016/0735-1097(90)90282-T.

24. Austen WG, Edwards JE, Frye RL, Gensini GG, Gott VL, Griffith LS, et al. A reporting system on patients evaluated for coronary artery disease. Report of the Ad Hoc Committee for Grading of Coronary Artery Disease, Council on Cardiovascular Surgery, American Heart Association. Circulation. 1975;51(4 Suppl):5-40. [PubMed: 1116248].

25. Niskanen L, Siitonen O, Suhonen M, Uusitupa MI. Medial artery calcification predicts cardiovascular mortality in patients with NIDDM Diabetes Care. 1994;17(11):1252-6. [PubMed: 7821163]

26. Lehto S, Niskanen L, Suhonen M, Ronnemaa T, Saikku P, Laakso M. Association between Chlamydia pneumoniae antibodies and intimal calcification in femoral arteries of nondiabetic patients. Arch Intern Med. 2002;162(5):594-9. [PubMed: 11871929].

27. Blacher J, Guerin AP, Pannier B, Marchais SJ, London GM. Arterial calcifications, arterial stiffness, and cardiovascular risk in end-stage renal disease. Hypertension. 2001;38(4):938-42. [PubMed: 11641313].

28. Mackey RH, Venkitachalam L, Sutton-Tyrrell K. Calcifications, arterial stiffness and atherosclerosis. Adv Cardiol. 2007;44:234-44. doi: 10.1159/000096744. [PubMed: 17075212].

29. Zagura M, Serg M, Kampus P, Zilmer M, Eha J, Unt E, et al. Aortic stiffness and vitamin $D$ are independent markers of aortic calcification in patients with peripheral arterial disease and in healthy subjects. Eur
J Vasc Endovasc Surg. 2011;42(5):689-95. doi: 10.1016/j.ejvs.2011.07.027. [PubMed: 21871824].

30. Duprez DA, De Buyzere MM, De Bruyne L, Clement DL, Cohn JN. Small and large artery elasticity indices in peripheral arterial occlusive disease (PAOD). Vasc Med. 2001;6(4):211-4. [PubMed: 11958385].

31. Kals J, Lieberg J, Kampus P, Zagura M, Eha J, Zilmer M. Prognostic impact of arterial stiffness in patients with symptomatic peripheral arterial disease. Eur J Vasc Endovasc Surg. 2014;48(3):308-15. doi: 10.1016/j.ejvs.2014.05.018. [PubMed: 24962743].

32. Chen Q, Smith CY, Bailey KR, Wennberg PW, Kullo IJ. Disease location is associated with survival in patients with peripheral arterial disease. J Am Heart Assoc. 2013;2(5):e000304. doi: 10.1161/JAHA.113.000304. [PubMed: 24145740].

33. Working Group of the STEOTMOSCAD, Reviewers for the EOTMOSCAD, S. E. C. Guidelines Committee. Comments on the 2013 ESC guidelines on the management of stable coronary artery disease. Rev Esp Cardiol (Engl Ed). 2014;67(2):80-6. doi: 10.1016/j.rec.2013.11.007. [PubMed: 24795113]

34. Task Force M, Montalescot G, Sechtem U, Achenbach S, Andreotti F, Arden C, et al. 2013 ESC guidelines on the management of stable coronary artery disease: the Task Force on the management of stable coronary artery disease of the European Society of Cardiology. Eur Heart J. 2013;34(38):2949-3003. doi:10.1093/eurheartj/eht296. [PubMed: 23996286].

35. Liu YC, Sun Z, Tsay PK, Chan T, Hsieh IC, Chen CC, et al. Significance of coronary calcification for prediction of coronary artery disease and cardiac events based on 64-slice coronary computed tomography angiography. Biomed Res Int. 2013;2013:472347. doi: 10.1155/2013/472347. [PubMed: 23586041]. 